\title{
A RARE CASE REPORT OF PYOGENIC LIVER ABSCESS CAUSED BY SALMONELLA ENTERITIDIS
}

\author{
Rajeshwari Prabhakar Rao ${ }^{1}$
}

${ }^{1}$ Associate Professor, Department of Microbiology, SIMS \& RC, Mukka, Surathkal, Mangalore.

\begin{abstract}
BACKGROUND

Salmonella enteritidis is one of the most important serovars transmitted from animals to man and a serovar most commonly reported worldwide. Although infection with S. enteritidis is limited to the intestinal tract, under certain circumstances it may cross the mucosal barrier, disseminate and get established as some localized infectious focus. Although cited as a very uncommon cause, S. enteritidis may involve the liver and evolve into an overt abscess. Pyogenic liver abscess by a gas forming organism like S. enteritidis usually follow a fulminant course. Associated morbidity and mortality is high unless immediate therapeutic interventions are initiated.
\end{abstract}

\section{KEYWORDS}

Salmonella enteritidis, Pyogenic Liver Abscess, Gas Forming Organisms.

HOW TO CITE THIS ARTICLE: Rao RP. A rare case report of pyogenic liver abscess caused by Salmonella Enteritidis. J. Evolution Med. Dent. Sci. 2016;5(32):1775-1776, DOI: $10.14260 /$ jemds/2016/418

\section{INTRODUCTION}

All tissues and organs are susceptible to Salmonella and may manifest in a variety of clinical entities depending upon the site of localization. Localization of systemic infections is usually associated with predisposing conditions such as malignancy, diabetes, sickle cell disease, immunosuppression, structural abnormalities, etc. Perhaps 1\% of enteric infections with non-typhoidal Salmonella result in bacteraemia.[1],[2] Pyogenic Liver Abscess (PLA) is one of the common liver pathologies and data from different sources place the incidence rate from 1.1 to $17.6 / 1,00,000$ individuals. ${ }^{[3]}$ Salmonella is one of the well-known causes of liver abscess.

At least four Salmonella serovars, viz. typhi, paratyphi A, Enteritidis and infantis have been identified as causative agents of liver abscess. Here, we report a case of liver abscess caused by Salmonella enterica serovar enteritidis in an immunocompetent male. This organism being prolific fermenter, produces a large amount of gas at the site of localization and contributes significantly to increased morbidity and mortality. Prompt diagnosis and immediate therapeutic intervention is required in minimizing the injury to the patient.

\section{CASE REPORT}

A 28-year-old male was admitted to the Medicine Department of our hospital with 1-week history of high-grade fever with chills and rigors, moderate abdominal pain and vomiting. Patient gave history of diarrhoea and pain abdomen about 1 month before reporting to the hospital. There was no history of diabetes or tuberculosis in the past.

On examination he was conscious, febrile (Temperature$39^{\circ} \mathrm{C}$ ), pulse rate was $160 / \mathrm{min}$ and blood pressure was $90 / 60$ mmHg. He had pallor. There was no

Financial or Other, Competing Interest: None.

Submission 27-02-2016, Peer Review 26-03-2016,

Acceptance 01-04-2016, Published 21-04-2016.

Corresponding Author:

Dr. Rajeshwari Prabhakar Rao,

Associate Professor,

Department of Microbiology,

SIMS \& RC, Mukka,

Surathkal, Mangalore.

E-mail:dr.rajeshwariprao@gmail.com

DOI: $10.14260 /$ jemds $/ 2016 / 418$ icterus. Per abdomen examination elicited tenderness in the right hypochondrium and epigastric region. Liver was palpable about $6 \mathrm{~cm}$ below the costal margin. Rest of the systemic examination was within normal limits. Routine haematological investigations revealed haemoglobin $8 \mathrm{~g} \%$, total leucocyte count-17,000/cumm (Polymorphs 75\%, Lymphocytes 20\%, Monocytes 2\%, Eosinophils 3\%) and platelet count of 2.2 lacs/cumm. Alkaline phosphatase was borderline elevated to $240 \mathrm{IU}$.

Other liver function tests, renal function values and serum electrolytes were within reference ranges. Serology for HIV, hepatitis B virus and hepatitis $C$ virus were nonreactive. Widal test and $\operatorname{IgM}$ leptospira were negative. Ultrasonography of the abdomen showed mild ascites and enlargement of the liver with features suggestive of abscess involving segment VI and VII, measuring $8.5 \mathrm{~cm}$ x $8.5 \mathrm{~cm} \times 8$ $\mathrm{cm}$ and volume $320 \mathrm{cc}$. The patient was empirically started on intravenous vancomycin, monocef and metronidazole. But patient developed respiratory distress and his condition deteriorated further. Respiratory examination showed decreased air entry and crepitations in the right lower lobe. X-ray chest showed right-sided pleural effusion. Contrast enhanced CT scan abdomen revealed a large liver abscess in the right lobe communicating with the pleural cavity of size $8.5 \mathrm{~cm} \times 8.5 \mathrm{~cm} \times 8 \mathrm{~cm}$. Ultrasound guided liver aspiration was done. About $35 \mathrm{~mL}$ of pus was aspirated and sent for microbiological investigations.

The pus sample was processed as per standard microbiological techniques. Wet mount of pus was negative for trophozoites of Entamoeba histolytica. Gram smear showed Gram-negative bacilli along with pus cells. ZiehlNeelsen stain was negative for acid fast bacilli. On blood agar, 2-3 mm diameter non-haemolytic colonies and on MacConkey agar, non-lactose fermenting colonies were obtained. The isolate was identified as S. enterica subspecies enterica serovar enteritidis on the basis of biochemical reactions and further confirmed by agglutination test with specific antisera.

It was found to be susceptible to ampicillin, cotrimoxazole, third generation cephalosporins and ciprofloxacin by Kirby Bauer disc diffusion method. Blood and urine cultures were sterile. No pathogenic organism was grown in stool culture. For pleural effusion, pleural tapping was done and intercostal drain was inserted. Pleural fluid 
also grew S. enteritidis with the antibiotic pattern compatible with the isolate from liver abscess. Treatment was changed accordingly. Metronidazole was stopped and ofloxacin added. However, the condition of the patient continued to deteriorate. He developed septicaemic shock and succumbed to his illness after 5 days of admission.

\section{DISCUSSION}

The three major forms of hepatic abscess based on aetiology are pyogenic, amoebic and fungal. Most common pathogens of the pyogenic hepatic abscesses are Escherichia coli, Klebsiella pneumoniae, Bacteroides, Enterococci, Streptococci and Staphylococci. ${ }^{[4]}$ Among gas forming PLA, K. pneumoniae is the most frequently cultured organism.[5] PLA due to Salmonella species is not that common and low incidence of hepatic manifestations may be explained by the phagocytic activity of its well-established reticuloendothelial system.

Soni et al. have also commented on the low frequency of abscess formation in Salmonella infections. Of all the serovars associated with hepatic abscess, Enteritidis is of special concern because of its potential to form gas from carbohydrates. It produces the formic hydrogen lyase, which converts formic acid a product of carbohydrate fermentation into $\mathrm{CO}_{2}$ and $\mathrm{H}_{2}$. The accumulated gas causes impaired transportation of gases and nutrients in the local tissues, promotes tissue destruction and results in abscess.[6] In our case, S. enteritidis which produces a large amount of gas was the causative agent of PLA. In such a case, mortality is as high as $27.7 \%$ when compared with the $14.4 \%$ mortality in nongas forming group.[5] Hence, prompt recognition and appropriate treatment is crucial for the management of patient.

Pyogenic liver abscess due to enteritidis have been reported with pre-existing hepatobiliary diseases such as cholelithiasis, amoebic abscess, echinococcal cysts, intrahepatic haematoma, hepatocellular carcinoma, etc.[7] However, in this case no such pre-existing condition was present. Furthermore, Salmonella liver abscess is mostly seen in the immunocompromised state, while our case was an immunocompetent adult male. Common presentations of PLA include abdominal pain, fever, chills, nausea and vomiting and a general feeling of illness. Blood culture was sterile in this case. The likely source of the liver abscess in our patient could be secondary to the seeding of infection from the transient portal bacteraemia or infection localized in the gall bladder could have travelled to the liver parenchyma. Pyogenic abscesses can be single or multiple.

But Salmonella abscesses like amoebic abscesses are predominantly solitary and located in the right lobe.[8] Ultrasonography and other imaging studies have wonderful sensitivities to pick up abscess pathologies, but microbiological diagnosis is absolutely essential to establish a causal relationship and strategizing further therapeutic plans.[9] Here, despite the fact that treatment was immediately modified to confirm to the antimicrobial sensitivity pattern of enteritidis, patient could not be saved probably because the patient had already landed into septicaemia.
Organisms like S. enteritidis, which restrict to the intestinal tract and behave in a predictable manner when affect extraintestinal sites like liver, may cause fatal damage as seen in this case. Such cases warrant the scientific community to take a relook at the treatment algorithms. Such cases also need to be diagnosed at the initial stage and be kept on surveillance for the development of morbid conditions like liver abscess. This is important in geographical areas where Salmonella infections are endemic.

Enteritidis is one of the rare causes of liver abscess. But in endemic geographical locations, this should be considered in the differential diagnosis, especially in cases with a proven history of intestinal illness. Liver abscess caused by gas forming organisms carry a high mortality and warrant immediate therapeutic intervention, which may include decisive surgical management and dedicated Intensive Care.

\section{REFERENCES}

1. Mahajan RK, Sharma S, Madan P, et al. Pyogenic liver abscess caused by salmonella enteritidis: a rare case report. Indian J Pathol Microbiol 2014;57(4):632-4.

2. Mandal BK, Brennand J. Bacteraemia in salmonellosis: a 15 year retrospective study from a regional infectious diseases unit. BMJ 1988;297:1242-3.

3. Tian LT, Yao K, Zhang XY, et al. Liver abscesses in adult patients with and without diabetes mellitus: an analysis of the clinical characteristics, features of the causative pathogens, outcomes and predictors of fatality: a report based on a large population, retrospective study in China. Clin Microbiol Infect 2012(9);18:E314-30.

4. Meddings L, Myers RP, Hubbard J, et al. A populationbased study of pyogenic liver abscesses in the united states: incidence, mortality, and temporal trends. Am J Gastroenterol 2010;105(1):117-24.

5. Chou FF, Sheen-Chen SM, Chen YS, et al. The comparison of clinical course and results of treatment between gasforming and non-gas-forming pyogenic liver abscess. Arch Surg 1995;130(4):401-5.

6. Lee HL, Lee HC, Guo HR, et al. Clinical significance and mechanism of gas formation of pyogenic liver abscess due to klebsiella pneumoniae. J Clin Microbiol 2004;42(6):2783-5.

7. Gomceli I, Gurer A, Ozdogan M, et al. Salmonella typhi abscess as a late complication of simple cyst of the liver: a case report. Turk J Gastroenterol 2006;17(2):151-2.

8. Vidal JE, Silva PR, Schiavon Nogueira R, et al. Liver abscess due to salmonella enteritidis in a returned traveler with HIV infection: case report and review of the literature. Rev Inst Med Trop Sao Paulo 2003;45(2):115-7.

9. Tulachan SS, Wilkins CE, Cutrona AF, et al. Hepatic abscess associated with salmonella serotype $B$ in a chronic alcoholic patient. Hepatobiliary Pancreat Dis Int 2013;12(4):440-2. 